\title{
Bryophytes of Libya. II. Musci: an annotated checklist
}

\author{
Wagieh E. El-Saadawi ${ }^{1}$, Hanaa M. Shabbara ${ }^{1}$, Said G. Youssef ${ }^{2 *}$, Manal I. Khalil ${ }^{1}$ \\ ${ }^{1}$ Botany Department, Faculty of Science, Ain Shams University, Cairo, Egypt \\ ${ }^{2}$ Botany Department, Faculty of Science, Benha University, Egypt \\ *Corresponding author. msmyes68@yahoo.com. Tel.: 201003250750
}

\begin{abstract}
A list is given for the, hitherto, known moss flora of Libya. It includes 102 taxa, belonging to 45 genera in 16 families and 8 orders under class Bryopsida, and their distribution in 20 surveyed localities. Significant annotations are given regarding distribution of the taxa in Libya, in the Mediterranean area, dominance and rarity, old and new records, other relevant information and a list of synonyms.
\end{abstract}

Keywords: Bryoflora, Checklist, Libya, Mosses

\section{Introduction}

The, up till now known, bryoflora of Libya consists of 131 taxa; of which 29 belong to Hepatophyta and 102 to Bryophyta (Musci). An annotated checklist of the Hepatophyta has been published quite recently (Youssef 2017). An annotated checklist of the Musci (mosses) remains, therefore, mostly needed.

Work on mosses of Libya started about one and a half century ago. The first was that of Ascherson (1881), followed by many others including Bottini (1914), Pampanini (1917, 1931), Rungby (1962), Ochi (1972), Bizot (1973), Arts et al. (1995) and Gallego et al. (1999) (see Ros et al 1999 for full list of references). Mosses reported, in these papers as occurring in Libya were complied, together with mosses reported from other NorthAfrican countries, in one long list published by Ros et al. in 1999. In that list 110 moss taxa were reported as occurring in Libya. Shabbara \& Ghanem (2006), Youssef et al. (2009a, b) added three new records to the moss flora of Libya but reduced the total number of mosses of this country to 107,108 and 109 respectively, however, more corrections and nomenclatural changes resulted in a further reduction to only 91 taxa as came in the more lengthy list (which included names of mosses of all Mediterranean countries) published in 2013, also by Ros et al.

Information given in Ros et al. (2013) shows that the report of the majority of the Libyan mosses (84 out of 91 taxa) were based on collections published before 1962, that more than one fourth of the Libyan mosses (25 taxa) represent single records (i.e. based on only one collection) and that several of the records are doubtful and require confirmation. This shows how far our knowledge of the main bulk of the moss flora of Libya is based on old collections and that the structure of this flora might be remarkably different at present necessitating, therefore, recent collection activities.

Mosses known from Libya are mainly reported from its north near the Mediterranean coast at: Tobruk, Darnah, Chersa, Al Qubbah, Mechili, Susa, Shahet (Cirene), Beida, Wadi Kouf, Tocra, Tecniz, Al Marj, Tolmetta, Benghazi, Tripoli, Borgo and Gharian (Fig. 1). There are three other moss collection localities namely Bosco Zorda, Wadi Balgader and Wadi Sambar mentioned in the literature; however we could not find them on available maps of Libya.

With the purpose of updating the moss flora of Libya on mined, S.G. Youssef (one of the authors of this paper) made recent moss collections, between the years 2004-2008, from five localities namely: Beida, Wadi Kouf, Shahet, Mas'sa and Hani'ya (Fig. 1); all belong to Al-Jabal Al-Akhdar region in the north east of Libya. Mas'sa and Hani'ya are two new localities not surveyed for mosses before these recent collections. Parts of the data on the mosses recently collected from Beida and Wadi Kouf have already been published (Shabbara \& Ghanem 2006; 


\section{Bryophytes of Libya. II. Musci: an annotated checklist}

Youssef et al. 2009 a, b; Youssef et al. 2017a,b and Khalil \& Youssef 2017) but data on the mosses of the two new localities
Mas'sa and Hani'ya in addition to Shahet have not been published yet.

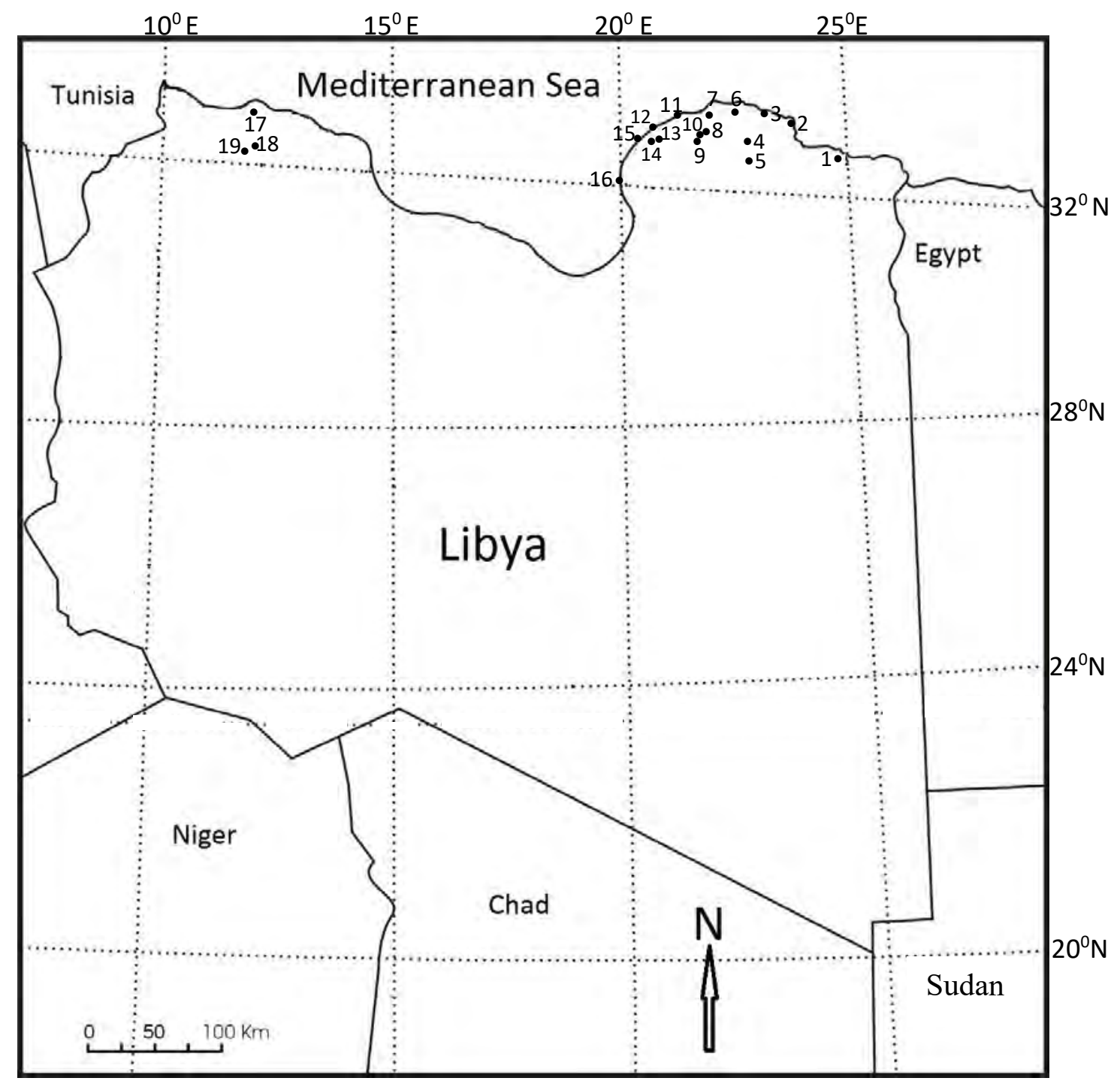

Fig. 1. Map showing sites of old and new collection of mosses in Libya. 1- Tobruk, 2- Darnah, 3- Chersa, 4- Al Qubbah, 5- Mechili, 6-Susa, 7- Shahet, 8- Beida, 9- Wadi Kouf, 10- Mas'sa, 11-Hani;ya, 12- Tocra, 13- Tecniz, 14- Al Marj, 15- Tolmetta 16- Benghazi, 17- Tripoli, 18- Borgo and 19- Gharian,

The part that has been, hitherto, published on these recent moss collections confirmed the existence of 25 of the old Libyan moss records (Youssef et al. 2017a, b and Khalil \& Youssef 2017) and added 14 new records to the bryoflora of Libya; 1 Fabroniaceae (Shabbara \& Ghanem, 2006), 2 Orthotrichacea (Youssef et al. 2009a, b), 7 Brachytheciaceae (Youssef et al. 2017a), 2 Pottiaceae (Youssef et al. 2017b) and 2 Bryaceae (Khalil \& Youssef 2017). The 3 species (1 Fabroniaceae and 2 Orthotrichacea) were included in the list of Ros et al. (2013) which maintained 91 taxa, while the other 11 species (7 Brachytheciaceae, 2 Pottiaceae and
2 Bryaceae) raised the total number of the mosses known from Libya to 102 taxa.

The present paper is the seventh in this series of recent publications on the Libyan moss flora and aims to present a more up to date and annotated moss list including the 102 taxa, hitherto known from Libya. It is worthy to mention that this is the first recent list confined to only Libyan mosses since earlier important lists were too old (Pampanini, 1931) or included the bryoflora of Libya as a part of wider geographical areas e.g. North Africa (Ros et al. 1999); Mediterranean area (Ros et al. 2013).

The 102 moss taxa included in the 
present list belong to 45 genera, 16 families, 8 orders, all under class Bryopsida. Names of the 8 orders and the 16 families and the number of taxa each includes are given in
Table 1, while the main list following Table 1 includes names of the 102 taxa, their families and their distribution in the 20 surveyed and published Libyan localities.

Table 1: Names of the 8 orders and 16 families representing the, up till now known, moss flora of Libya and the number of genera and taxa under each.

\begin{tabular}{|c|c|c|c|c|}
\hline Order & No. of Taxa & Family & Genera & No. of Taxa \\
\hline Pottiales & 43 & Pottiaceae & 16 & 43 \\
\hline \multirow{5}{*}{ Hypnales } & \multirow{5}{*}{22} & Amblystegiaceae & 1 & 1 \\
\hline & & Brachytheciaceae & 10 & 18 \\
\hline & & Fabroniaceae & 1 & 1 \\
\hline & & Hypnaceae & 1 & 1 \\
\hline & & Leptodontaceae & 1 & 1 \\
\hline \multirow[t]{2}{*}{ Bryales } & \multirow[t]{2}{*}{13} & Bryaceae & 2 & 11 \\
\hline & & Mniaceae & 1 & 2 \\
\hline \multirow{3}{*}{ Dicranales } & \multirow{3}{*}{9} & Dicranaceae & 1 & 2 \\
\hline & & Ditrichaceae & 2 & 2 \\
\hline & & Fissidentaceae & 1 & 5 \\
\hline Funariales & 5 & Funariaceae & 3 & 5 \\
\hline Orthotrichales & 4 & Orthotrichaceae & 2 & 4 \\
\hline \multirow[t]{2}{*}{ Grimmiales } & \multirow[t]{2}{*}{4} & Grimmiaceae, & 1 & 3 \\
\hline & & Ptychomitriaceae & 1 & 1 \\
\hline \multirow[t]{2}{*}{ Encalyptales } & 2 & Encalyptaceae & 1 & 2 \\
\hline & 102 & & 45 & 102 \\
\hline
\end{tabular}

\section{List of Mosses}

Distribution of the 102 moss taxa in the 20 surveyed and published localities of Libya based on 1Bottini, 1914; 2- Pampaini, 1917; 3- Pampaini, 1931; 4- Bizot, 1973; 5- Shabbara \& Ghanem, 2006; 6- Ros et al., 2013; 7- Youssef et al., 2009a; 8- Youssef et al., 2009b; 9- Youssef et al., 2017a; 10Youssef et al., 2017b and11- Khalil \& Youssef, 2017. Taxa are arranged alphabetically and referred to their families. Abbreviations: Tob $=$ Tobruk, Dar $=$ Darnah, Che $=$ Chersa, $Q u b=A 1$ Qubbah, Mec $=$ Mechili, Sus $=$ Susa, Sha $=$ Shahet, Bda $=$ Beida, WKf $=$ Wadi Kouf, Toc $=$ Tocra, Tec $=$ Tecniz, Mrj $=$ Al Marj, Tol $=$ Tolmetta, Ben $=$ Benghazi, Trp $=$ Tripoli, Bor $=$ Borgo, Ghn $=$ Gharian, BoZ $=$ Bosco Zorda, $\mathrm{WBl}=$ Wadi Balgader and WSm $=$ Wadi Sambar.

\section{Symbols}

o Report based on collections published before 1962.

- $\quad$ Report based on at least one collection published during or after 1962 and before 2013.

(o) Doubtful report based on collections published before 1962.

$\square \quad$ Recorded after 2013, but also earlier.

Recorded only after 2013

$\diamond \quad$ Reported without reference to any locality (Only Libya).

? Number of localities not known because available publications mentioned only Libya. 


\section{Bryophytes of Libya. II. Musci: an annotated checklist}

\begin{tabular}{|c|c|c|c|c|c|c|c|c|c|c|c|c|c|c|c|c|c|c|c|c|c|c|}
\hline Taxa $\quad$ Localities & Tob & Dar & Che & Qub & Mec & Sus & Sha & Bda & WKf & Toc & Tec & Mrj & Tol & Ben & $\operatorname{Trp}$ & Bor & Ghn & BoZ & WBl & WSm & $\begin{array}{l}\text { Only } \\
\text { Libya }\end{array}$ & $\begin{array}{l}\text { Total no. } \\
\text { of } \\
\text { localities }\end{array}$ \\
\hline \multicolumn{23}{|l|}{ Acaulon Müll.Hal. (Pottiaceae) } \\
\hline triquetrum (Spruce) Müll.Hal. & & & & & & & & & & & & & & $\circ 2, \circ 3$ & & & & & & & & 1 \\
\hline \multicolumn{23}{|l|}{ Aloina Kindb. (Pottiaceae) } \\
\hline ambigua (Bruch \& Schimp.) Limpr. & & $\circ 3$ & & & & & & & & & $\circ 3$ & $\circ 3$ & $\circ 3$ & $\circ 3$ & $01, \bullet 4$ & & & & & & & 6 \\
\hline bifrons (De Not.) Delgad. & & & & & $\circ 3$ & & & & & & & & & & & & & & & & & 1 \\
\hline rigida (Hedw.) Limpr. & & & & & & & & $\square 10$ & & & & $\circ 3$ & & $\circ 2, \circ 3$ & $\circ 1$ & & $\circ 1$ & & & & & 5 \\
\hline \multicolumn{23}{|l|}{ Barbula Hedw. (Pottiaceae) } \\
\hline bolleana (Müll.Hal.) Broth. & & & & & & & & & & & & & & & & & & & & & $\diamond, 06$ & $?$ \\
\hline convoluta Hedw. & & & & & & & & $\square 11$ & & & & & & 02,03 & & & & & & & & 2 \\
\hline unguiculata Hedw. & & $\circ 3$ & & & & & & & $\circ 3$ & & $\circ 3$ & $\circ 3$ & & & & & & $\circ 3$ & & & & 5 \\
\hline \multicolumn{23}{|l|}{\begin{tabular}{|lll}
$\begin{array}{l}\text { Brachythecium } \\
\text { ciaceae) }\end{array}$ & Schimp. & (Brachythe- \\
\end{tabular}} \\
\hline rutabulum (Hedw.) Schimp & & & & & & & & & 49 & & & & & & & & & & & & & 1 \\
\hline $\begin{array}{l}\text { salebrosum (Hoffm. ex F.Weber \& } \\
\text { D.Mohr) Schimp. } \\
\end{array}$ & & & & & & & $\circ 3$ & & & & & & & & & & & & & & & 1 \\
\hline \multicolumn{23}{|l|}{ Bryum Hedw. (Bryaceae) } \\
\hline argenteum Hedw. & & & & & & & & $\square 11$ & & & & $\circ 3$ & & & & & & $\circ 3$ & & & & 3 \\
\hline canariense Brid. & & $\circ 3$ & & & & & & & & & & & & & & & & & & & & 1 \\
\hline dichotomum Hedw. & & $\circ 3$ & & $\circ 3$ & $\circ 3$ & & $\circ 3$ & $\square 11$ & & & $\circ 3$ & $\circ 3$ & $\circ 3$ & $\circ 2, \circ 3$ & $\circ 1$ & & $\circ 1$ & $\circ 3$ & & $\circ 3$ & & 13 \\
\hline radiculosum Brid. & & $\circ 3$ & & & & & $\circ 3$ & $\square 11$ & $\circ 3$ & & & $\circ 3$ & & & & $\circ 3$ & & $\circ 3$ & $\circ 3$ & & & 8 \\
\hline valparaisense Thér. & & & & & & & & & & & & & & & & & & & & & $\diamond, \cdot 6$ & $?$ \\
\hline \multicolumn{23}{|l|}{\begin{tabular}{|lll} 
Campylostelium Bruch & $\&$ & Schimp. \\
(Ptychomitriaceae)
\end{tabular}} \\
\hline pitardii (Corb.) E.Maier & & & & & & & & & & & & & & & & & & & & & $\diamond, \circ 6$ & $?$ \\
\hline \multicolumn{23}{|l|}{ Cheilothela Broth. (Ditrichaceae) } \\
\hline chloropus (Brid.) Broth. & & & & $\circ 3$ & & & & & & & & & & & & & & $\circ 3$ & & $\circ 3$ & & 3 \\
\hline \multicolumn{23}{|l|}{ Crossidium Jur. (Pottiaceae) } \\
\hline crassinervium (De Not.) Jur. & & & & & & & & $\square 10$ & & & & & & & & & & & & & $\diamond, \circ 6$ & $1+?$ \\
\hline squamiferum (Viv.) Jur. & & & & & $\circ 3$ & & & $\begin{array}{l}\square 10, \\
\square 11\end{array}$ & & & $\circ 3$ & $\circ 3$ & & & $\circ 1$ & & $\circ 1$ & $\circ 3$ & & & & 7 \\
\hline $\begin{array}{l}\text { squamiferum (Viv.) Jur. var. pottioide- } \\
\text { um (De Not.) Mönk. }\end{array}$ & & & & & & & & $\square 10$ & & & & & & & & & & & & & $\diamond, \circ 6$ & $1+?$ \\
\hline \multicolumn{23}{|l|}{$\begin{array}{l}\text { Dicranella (Müll.Hal.) Schimp. (Dicra- } \\
\text { naceae) }\end{array}$} \\
\hline howei Renauld \& Cardot & & & & & & $\circ 3$ & & & & & & & & & & & & & & & & 1 \\
\hline varia (Hedw.) Schimp. & & & & & & & & & $\circ 3$ & & $\circ 3$ & & & & $\cdot 4$ & & & & & & & 3 \\
\hline
\end{tabular}


Wagieh E. El-Saadawi et al.

\begin{tabular}{|c|c|c|c|c|c|c|c|c|c|c|c|c|c|c|c|c|c|c|c|c|c|c|}
\hline Taxa $\quad$ Localities & Tob & Dar & Che & Qub & Mec & Sus & Sha & Bda & WKf & Toc & $\mathrm{Tec}$ & Mrj & Tol & Ben & Trp & Bor & Ghn & BoZ & WB1 & WSm & $\begin{array}{l}\text { Only } \\
\text { Libya }\end{array}$ & $\begin{array}{l}\text { Total no. } \\
\text { of } \\
\text { localities }\end{array}$ \\
\hline \multicolumn{23}{|l|}{ Didymodon Hedw. (Pottiaceae) } \\
\hline acutus (Brid.) K.Saito & & & & $\circ 3$ & & & & $\square 10$ & & & & & & & & & & & & & & 2 \\
\hline fallax (Hedw.) R.H.Zander & & & & & & & & $\square 10$ & & & & & & & & & $\circ 1$ & & & $\circ 3$ & & 3 \\
\hline insulanus (De Not.) M.O.Hill & & & & & & & & $\square 10$ & & & & & & & & & $\circ 1$ & & & & & 2 \\
\hline luridus Hornsch. & & $\circ 3$ & & & & & $\circ 3$ & $\square 10$ & $\circ 3$ & & $\circ 3$ & $\circ 3$ & $\circ 3$ & & & & $\circ 1$ & $\circ 3$ & & & & 9 \\
\hline rigidulus Hedw. & & $\circ 3$ & & & & $\circ 3$ & $\circ 3$ & & & & & & & & $\bullet 4$ & & & & & & & 4 \\
\hline tophaceus (Brid.) Lisa & & $\circ 3$ & & & & $\circ 3$ & $\circ 3$ & & $\circ 3$ & 03 & & & & & $01, \bullet 4$ & & $\circ 1$ & & & & & 7 \\
\hline vinealis (Brid.) R.H.Zander & & & & & & & ○3 & \begin{tabular}{|l|}
$1, \circ 3$ \\
$\square 10, \square 11$ \\
\end{tabular} & & & & & & $\circ 2, \circ 3$ & & & $\circ 1$ & & & $\circ 3$ & & 5 \\
\hline \multicolumn{23}{|l|}{$\begin{array}{l}\text { Distichium Bruch \& Schimp. (Ditricha- } \\
\text { ceae) }\end{array}$} \\
\hline capillaceum (Hedw.) Bruch \& Schimp. & & & & & & & & & & & & & & & & & & & & & $\diamond, \circ 6$ & $?$ \\
\hline \multicolumn{23}{|l|}{$\begin{array}{l}\text { Drepanocladus (Müll.Hal.) G.Roth } \\
\text { (Amblystegiaceae) }\end{array}$} \\
\hline aduncus (Hedw.) Warnst. & & $(0) 3$ & & & & & & & & & & & & & & & & & & & & 1 \\
\hline \multicolumn{23}{|l|}{ Encalypta Hedw. (Encalyptaceae) } \\
\hline $\begin{array}{l}\text { rhaptocarpa Schwägr. var. leptodon } \\
\text { Lindb. }\end{array}$ & & & & & & & & & $\circ 3$ & & & & & & & & $\circ 1$ & & & & & 2 \\
\hline vulgaris Hedw. & & & & & & & $\circ 3$ & & & & & & & & & & & & & & & 1 \\
\hline \multicolumn{23}{|l|}{ Entosthodon Schwägr.(Funariaceae) } \\
\hline angustifolius Jur. \& Milde & $\circ 3$ & & & & & & & & & & & & & & & & & & & & & 1 \\
\hline duriaei Mont. & & & & & & & & & & & & & & & & & & & & & $\diamond, \circ 6$ & $?$ \\
\hline muhlenbergii (Turner) Fife & & $\circ 3$ & & $\circ 3$ & & & & & & & & & & $\circ 2, \circ 3$ & & & & & & & & 3 \\
\hline \multicolumn{23}{|l|}{$\begin{array}{l}\text { Eucladium Bruch \& Schimp. (Pottiace- } \\
\text { ae) }\end{array}$} \\
\hline verticillatum (With.) Bruch \& Schimp. & & $\circ 3$ & & & & $\circ 3$ & & $\square 10$ & $\circ 3$ & & & & & & & & & & $\circ 3$ & & & 5 \\
\hline \multicolumn{23}{|l|}{$\begin{array}{l}\text { Eurhynchiastrum Ignatov } \\
\text { \& Huttunen (Brachytheciaceae) }\end{array}$} \\
\hline \begin{tabular}{|llll}
$\begin{array}{l}\text { pulchellum } \\
\text { Huttunen }\end{array}$ & (Hedw.) Ignatov $\&$ \\
\end{tabular} & & & & & & & & & 49 & & & & & & & & & & & & & 1 \\
\hline \multicolumn{23}{|l|}{ Fabronia Raddi (Fabroniaceae) } \\
\hline pusilla Raddi & & & & & & & & & $\cdot 5$ & & & & & & & & & & & & & 1 \\
\hline \multicolumn{23}{|l|}{ Fissidens Hedw. (Fissidentaceae) } \\
\hline $\begin{array}{l}\text { crassipes Wilson ex Bruch \& Schimp. } \\
\text { subsp. warnstorfii (M.Fleisch) Brugg.- } \\
\text { Nann. }\end{array}$ & & & & & & & & & & & & & & & & & & & & & $\diamond, \circ 6$ & $?$ \\
\hline crispus Mont. & $\circ 3$ & & & & & & & & & & & & & & & & & & & & & 1 \\
\hline pusillus (Wilson) Milde & & & & & & & $\circ 3$ & & & & & $\circ 3$ & & & & & & & $\circ 3$ & & & 3 \\
\hline rufulus Bruch \& Schimp. & & $\circ 3$ & & & & & & & & & & & & & & & & & & & & 1 \\
\hline
\end{tabular}




\section{Bryophytes of Libya. II. Musci: an annotated checklist}

\begin{tabular}{|c|c|c|c|c|c|c|c|c|c|c|c|c|c|c|c|c|c|c|c|c|c|c|}
\hline Localities & Tob & Dar & Che & Qub & Mec & Sus & Sha & Bda & WKf & Toc & Tec & Mrj & Tol & Ben & $\operatorname{Trp}$ & Bor & Ghn & BoZ & WB1 & WSm & $\begin{array}{l}\text { Only } \\
\text { Libya }\end{array}$ & $\begin{array}{l}\text { Total no. } \\
\text { of } \\
\text { localities }\end{array}$ \\
\hline $\begin{array}{l}\text { viridulus (Sw. ex Anon.) Wahlenb. var. } \\
\text { incurvus (Starke ex Röhl.)Waldh. }\end{array}$ & & & & & & & & $\circ 1, \circ 3$ & & & & & & & & & & & & & & 1 \\
\hline \multicolumn{23}{|l|}{ Funaria Hedw. (Funariaceae) } \\
\hline hygrometrica Hedw. & & & & & & $\circ 3$ & $\circ 3$ & & & & & $\circ 3$ & & & & & & & & & & 3 \\
\hline \multicolumn{23}{|l|}{ Funariella Sérgio (Funariaceae) } \\
\hline curviseta (Schwägr.) Sérgio & & & & & & & & & & & & & & & & & & & & & $\diamond, \circ 6$ & $?$ \\
\hline \multicolumn{23}{|l|}{ Grimmia Hedw. (Grimmiaceae) } \\
\hline lisae De Not. & & & & & & & & & $\circ 3$ & & & $\circ 3$ & $\circ 3$ & & & & & & & & & 3 \\
\hline orbicularis Bruch ex Wilson & & & & & & & & & $\circ 3$ & & & & & & & & $\circ 1$ & & & & & 2 \\
\hline pulvinata (Hedw.) Sm. & & & & & & & $\circ 3$ & & & & & & & & & & & & & & & 1 \\
\hline \multicolumn{23}{|l|}{$\begin{array}{l}\text { Gymnostomum Nees \& Hornsch. (Pot- } \\
\text { tiaceae) }\end{array}$} \\
\hline calcareum Nees \& Hornsch. & & $\circ 3$ & & & & 03 & & & & & & $\circ 3$ & & & & & & & $\circ 3$ & $\circ 3$ & & 5 \\
\hline \multicolumn{23}{|l|}{ Gyroweisia Schimp. (Pottiaceae) } \\
\hline reflexa (Brid.) Schimp. & & o3 & & & & & & & & & & & & & & & & & & & & 1 \\
\hline \multicolumn{23}{|l|}{$\begin{array}{l}\begin{array}{l}\text { Homalothecium Schimp. (Brachythe- } \\
\text { ciaceae) }\end{array} \\
\end{array}$} \\
\hline aureum (Spruce) H.Rob. & & & & & & & $\circ 3$ & & & & & $\circ 3$ & & & & & & & & & & 2 \\
\hline philippeanum (Spruce) Schimp. & & & & & & & & & $\circ 3$ & & & $\circ 3$ & & & & & & & & & & 2 \\
\hline sericeum (Hedw.) Schimp. & & & & & & & & & $\square 9$ & & & & & & & & & & $\circ 3$ & 03 & & 3 \\
\hline \multicolumn{23}{|l|}{ Hypnum Hedw. (Hypnaceae) } \\
\hline $\begin{array}{l}\text { cupressiforme Hedw. var. lacunosum } \\
\text { Brid. }\end{array}$ & & & & & & & & & $\circ 3$ & & & & & & & & & & & & & 1 \\
\hline \multicolumn{23}{|l|}{ Leptodon D.Mohr (Leptodontaceae) } \\
\hline smithii (Hedw.) F.Weber \& D.Mohr & & & & & & & & & $\circ 3$ & & & & & & & & & & & & & 1 \\
\hline \multicolumn{23}{|l|}{ Microbryum Schimp. (Pottiaceae) } \\
\hline davallianum (Sm.) R.H.Zander & & $\circ 3$ & & & & & & $\square 10$ & & & & & & & & & & & & & & 2 \\
\hline rectum (With.) R.H.Zander & & & & & & & & 410 & & & & & & & & & & & & & & 1 \\
\hline starckeanum (Hedw.) R.H.Zander & & $\circ 3$ & & $\circ 3$ & & & & $\begin{array}{l}\square 10 \\
\square 11\end{array}$ & & & & & & $\circ 2, \circ 3$ & & & & & & & & 4 \\
\hline \multicolumn{23}{|l|}{$\begin{array}{l}\text { Microeurhynchium Ignatov \& Vanderp. } \\
\text { (Brachytheciaceae) }\end{array}$} \\
\hline pumilum (Wilson) Ignatov \& Vanderp. & & & & & & & & & & & & $\circ 3$ & & & & & & & & & & 1 \\
\hline \multicolumn{23}{|l|}{ Orthotrichum Hedw. (Orthotrichaceae) } \\
\hline anomalum Hedw. & & & & & & & & & & & & & & & & & & & & & $\diamond, \circ 6$ & $?$ \\
\hline diaphanum Schrad. ex Brid. & & & & & & & & & $\cdot 8$ & & $\circ 3$ & $\circ 3$ & & & & & & & & & & 3 \\
\hline schimperi Hammar & & & & & & & & & $\cdot 8$ & & & & & & & & & & & & & 1 \\
\hline
\end{tabular}


Wagieh E. El-Saadawi et al.

\begin{tabular}{|c|c|c|c|c|c|c|c|c|c|c|c|c|c|c|c|c|c|c|c|c|c|c|}
\hline Localities & Tob & Dar & Che & Qub & Mec & Sus & Sha & Bda & WKf & Toc & $\mathrm{Tec}$ & Mrj & Tol & Ben & $\operatorname{Trp}$ & Bor & Ghn & BoZ & WB1 & WSm & $\begin{array}{l}\text { Only } \\
\text { Libya }\end{array}$ & $\begin{array}{c}\text { Total no. } \\
\text { of } \\
\text { localities }\end{array}$ \\
\hline \multicolumn{23}{|l|}{ Pohlia Hedw. (Mniaceae) } \\
\hline atropurpurea (Wahlenb.) H.Lindb. & & & & & & & & & & & & & & & & & & & & & $\diamond, \circ 6$ & $?$ \\
\hline melanodon (Brid.) A.J.Shaw & & & & & & & & & & & & & & & & & & & & & $\diamond, \circ 6$ & $?$ \\
\hline \multicolumn{23}{|l|}{$\begin{array}{l}\begin{array}{l}\text { Pseudocrossidium } \\
\text { tiaceae) }\end{array} \\
\end{array}$} \\
\hline $\begin{array}{l}\text { hornschuchianum } \\
\text { R.H.Zander }\end{array}$ & & $\circ 3$ & & & & & & $\square 10$ & & & & & & & & & ০1 & $\circ 3$ & & & & 4 \\
\hline revolutum (Brid.) R.H.Zander & & & & & & & $\circ 3$ & $\circ 1, \circ 3$ & & & & & & & & & & $\circ 3$ & & & & 3 \\
\hline \multicolumn{23}{|l|}{$\begin{array}{l}\text { Pseudoscleropodium (Brachytheciace- } \\
\text { ae) }\end{array}$} \\
\hline purum (Hedw.) M.Fleisch & & & & & & & & & 49 & & & & & & & & & & & & & 1 \\
\hline \multicolumn{23}{|l|}{ Ptychostomum Hornsch. (Bryaceae) } \\
\hline $\begin{array}{l}\text { archangelicum (Bruch \& Schimp.) } \\
\text { J.R.Spence }\end{array}$ & & & & & & & & $<11$ & & & & & & & & & & & & & & 1 \\
\hline $\begin{array}{l}\text { boreale (F.Weber \& D.Mohr) Ochyra } \\
\text { \& Bednarek-Ochyra }\end{array}$ & & $\circ 3$ & & & & & & & & & & $\circ 3$ & & & & & & & & & & 2 \\
\hline $\begin{array}{llll}\text { capillare } & \text { (Hedw.) } & \text { Holyoak } & \& \\
\text { N.Pedersen } & & & \\
\end{array}$ & & $\circ 3$ & & & & & & & & & $\circ 3$ & & & & & & & & & $\circ 3$ & & 3 \\
\hline $\begin{array}{ccc}\text { donianum } & \text { (Grev.) } & \text { Holyoak\& } \\
\text { N.Pedersen } & & \\
\end{array}$ & & & & & & $\circ 3$ & & & & & & & & & & & & & & & & 1 \\
\hline $\begin{array}{l}\text { imbricatulum (Müll.Hal.) Holyoak \& } \\
\text { N.Pedersen }\end{array}$ & & & & & & & & $\varangle 11$ & & & & & & & & & & & & & & 1 \\
\hline $\begin{array}{l}\text { torquescens (Bruch \& Schimp.( Ros \& } \\
\text { Mazimpaka }\end{array}$ & & & & & & & & & & & & & & & $\circ 1$ & & & $\circ 3$ & & & & 2 \\
\hline \multicolumn{23}{|l|}{$\begin{array}{l}\text { Rhynchostegiella (Schimp.) Limpr. } \\
\text { (Brachytheciaceae) }\end{array}$} \\
\hline curviseta (Brid.) Lindb. & & & & & & & & & & & & & & & & & & & & $\circ 3$ & & 1 \\
\hline litorea (De Not.) Limpr. & & & & & & & & & & & & & & & & & & & & $\circ 3$ & & 1 \\
\hline \multicolumn{23}{|l|}{$\begin{array}{l}\text { Rhynchostegium Schimp. (Brachythe- } \\
\text { ciaceae) }\end{array}$} \\
\hline confertum (Dicks.) Schimp. & & & & & & & $\circ 3$ & & & & & & & & & & & & ○3 & & & 2 \\
\hline $\begin{array}{l}\text { megapolitanum (Blandow ex F.Weber } \\
\text { \& D.Mohr) Schimp. }\end{array}$ & & & & & & & & & 49 & & & & & & & & & & & & & 1 \\
\hline riparioides (Hedw.) Cardot & & $\circ 3$ & & & & $\circ 3$ & & & $\square 9$ & & & & & & & & & & & & & 3 \\
\hline \multicolumn{23}{|l|}{\begin{tabular}{|lll}
$\begin{array}{l}\text { Sciuro-hypnum } \\
\text { ciaceae) }\end{array}$ & Hampe (Brachythe- \\
\end{tabular}} \\
\hline $\begin{array}{llll}\begin{array}{l}\text { plumosum } \\
\text { Huttunen }\end{array} & \text { (Hedw.) } & \text { Ignatov } & \& \\
\end{array}$ & & & & & & & & & 49 & & & & & & & & & & & & & 1 \\
\hline populeum (Hedw.) Ignatov \& Huttunen & & & & & & & & & & & & & & & & & & & o3 & & & 1 \\
\hline
\end{tabular}




\section{Bryophytes of Libya. II. Musci: an annotated checklist}

\begin{tabular}{|c|c|c|c|c|c|c|c|c|c|c|c|c|c|c|c|c|c|c|c|c|c|c|}
\hline Taxa $\quad$ Localities & Tob & Dar & Che & Qub & Mec & Sus & Sha & Bda & WKf & Toc & Tec & Mrj & Tol & Ben & $\operatorname{Trp}$ & Bor & Ghn & $\mathrm{BoZ}$ & WB1 & WSm & $\begin{array}{l}\text { Only } \\
\text { Libya }\end{array}$ & $\begin{array}{l}\text { Total no. } \\
\text { of } \\
\text { localities }\end{array}$ \\
\hline \multicolumn{23}{|l|}{$\begin{array}{l}\text { Scleropodium Bruch \& Schimp. (Brachythe- } \\
\text { ciaceae) }\end{array}$} \\
\hline tourretii (Brid.) L.F. Koch & & & & & & & & & 49 & & & & & & & & & & & & & 1 \\
\hline \multicolumn{23}{|l|}{ Scorpiurium Schimp (Brachytheciaceae) } \\
\hline circinatum (Bruch) M.Fleisch.\& Loeske & & $\circ 3$ & & & & & $\circ 3$ & $\circ 1, \circ 3$ & $\begin{array}{l}3, \\
\square 9\end{array}$ & & & & & & & & & & $\circ 3$ & $\circ 3$ & & 6 \\
\hline deflexifolium (Solms) M.Fleisch. \& Loeske & & & & & & & & & 49 & & & & & & & & & & & & & 1 \\
\hline \multicolumn{23}{|l|}{ Syntrichia Brid. (Pottiaceae) } \\
\hline laevipila Brid. & & & & & & & & & & & 03 & & & & & & & & & & & 1 \\
\hline montana Nees & & & & & & & $\circ 3$ & & 03 & & & $\circ 3$ & & & & & & & & & & 3 \\
\hline \multicolumn{23}{|l|}{ Timmiella (De Not.) Limpr. (Pottiaceae) } \\
\hline barbuloides (Brid.) Mönk. & 03 & & & & & & & $\square 10$ & & $\circ 3$ & & 03 & & & & & & & & 03 & & 5 \\
\hline \multicolumn{23}{|l|}{ Tortella (Müll.Hal.) Limpr. (Pottiaceae) } \\
\hline flavovirens (Bruch) Broth. & & & & & & & $\circ 3$ & & 03 & & & $\circ 3$ & & & & & & & & & & 3 \\
\hline inflexa (Bruch) Broth. & & & & & & & & 01,03 & & & & & & & & & & & & & & 1 \\
\hline nitida (Lindb.) Broth. & 03 & & 03 & & & & & & 03 & & & & & & & & & & 03 & 03 & & 5 \\
\hline squarrosa (Brid.) Limpr. & & & & & & & 03 & & & & & $\circ 3$ & & & & & & & & & & 2 \\
\hline tortuosa (Hedw.) Limpr. & & & & & & & & $\square 10$ & & & & & & & & & & & & & $\diamond, \circ 6$ & $1+?$ \\
\hline \multicolumn{23}{|l|}{ Tortula Hedw. (Pottiaceae) } \\
\hline $\begin{array}{l}\text { acaulon (With.) R.H.Zander var. pilif era } \\
\text { (Hedw). R.H.Zander }\end{array}$ & & 03 & & & & & & & & & & & & & & & & & & & & 1 \\
\hline atrovirens $(\mathrm{Sm}$.$) Lindb.$ & $\circ 3$ & & & & & & & & & & & & & & & & & & & & & 1 \\
\hline canescens Mont. & & & & & & & & & & & & & & & & & & & & & $\diamond, 06$ & $?$ \\
\hline marginata (Bruch \& Schimp) Spruce & $\circ 3$ & $\circ 3$ & & & & $\circ 3$ & & $\begin{array}{r}\circ 1, \circ 3 \\
\square 10\end{array}$ & & & & & & & & & & & 03 & & & 5 \\
\hline muralis Hedw. & ○3 & $\circ 3$ & & & & & $\circ 3$ & $\begin{array}{r}\circ 1, \circ 3 \\
\square 10\end{array}$ & & & o3 & $\circ 3$ & & & $\circ 1$ & $\circ 3$ & & $\circ 3$ & & & & 9 \\
\hline revolvens (Schimp.) G.Roth & & 03 & & 03 & & & & & & & 03 & 03 & & & & & $\circ 1$ & 03 & & & & 6 \\
\hline \multicolumn{23}{|l|}{ Trichostomum Bruch (Pottiaceae) } \\
\hline brachydontium Bruch & & & & & & & & $\square 10$ & & & & & & & & & & & & & $\diamond, \circ 6$ & $1+?$ \\
\hline crispulum Bruch & & 03 & & 03 & & & & & 03 & $\circ 3$ & 03 & 03 & & & & & $\circ 1$ & 03 & & & & 8 \\
\hline \multicolumn{23}{|l|}{ Weissia Hedw. (Pottiaceae) } \\
\hline condensa (Voit) Lindb. & & & & & & & & & & & & & & & & & & & & & $\diamond, 06$ & $?$ \\
\hline $\begin{array}{l}\text { controversa var. crispata (Nees and } \\
\text { Hornsch.) Nyholm }\end{array}$ & & & & & & & & 410 & & & & & & & & & & & & & & 1 \\
\hline \multicolumn{23}{|l|}{ Zygodon Hook. \& Taylor (Orthotrichaceae) } \\
\hline $\begin{array}{l}\text { catarinoi C.Garcia, F.Lara, Sérgio \& Sim- } \\
\text { Sim }\end{array}$ & & & & & & & & & .7 & & & & & & & & & & & & & 1 \\
\hline Number of taxa / locality & 7 & 26 & 1 & 7 & 3 & 9 & 19 & 30 & 30 & 3 & 12 & 24 & 4 & 8 & 9 & 2 & 13 & 13 & 10 & 12 & 16 & \\
\hline
\end{tabular}




\section{Annotations}

A- Based on Table 1 above, the following annotations were listed.

1- The 8 orders can be arranged in a descending order regarding the number of taxa representing each as follows: Pottiales (43), Hypnales (22), Bryales (13), Dicranales (9), Funariales (5), Grimmiales (4), Orthotrichales (4) and Encalyptales (2).

2- The largest family is Pottiaceae with 43 taxa followed by Brachytheciaceae with 18 taxa and Bryaceae with 11 taxa.

B- Based on the above list, the following annotations were listed.

1- This list is the first to show updated distribution of mosses in different Libyan localities.

2- The richest localities in moss species are: Darnah, Beida,Wadi Kouf and Al Marj, (over 20 species each) while the least are Chersa, Mechili, Tocra, and Borgo (under 4 species each). However, this is a temporal arrangement because most of these localities are under surveyed for mosses.

3- The 25 taxa that are recorded "after 2013, but also earlier" came mainly from Beida; only 3 of them came from Wadi Kouf.

4- Bryum dichotomum is the most widespread species in Libya, being recorded in 13 out of 20 surveyed localities, followed by Didymodon luridus and Tortula muralis in 9 localities each, Bryum radiculosum and Trichostomum crispulum in 8 localites each, Crossidium squamiferum and Didymodon tophaceus in 7 localities each, $\mathrm{Al}$ oina ambigua, Scorpiurium circinatum and Tortula revolvens in 6 localities each.

5- Forty-nine out of the 102 moss taxa of Libya, can be considered rare being recorded in only one or two localities in addition to the 16 taxa that were not referred to any particular locality but cited only as Libya, because they were not reported again from Libya except four of them (all from Beida) i.e. a total of 65 taxa are rare (more than $60 \%$ of the flora).

6- More than $50 \%$ of the taxa of Libya have been reported before 1962 and not again.

\section{Other annotations}

1-Only five taxa, namely: Bryum argenteum, B. dichotomum, Funaria hygrometrica, Tortula muralis and Trichostomum crispulum exist in all 34 Mediterranean countries and islands whereas 93 exist in 33-10 of these countries and islands. Only four taxa namely: Bryum valparaisense, Encalypta rhaptocarpa var. leptodon, Entosthodon angustifolius, and Zygodon catarinoi exist in less than 10 of these countries and islands (see Ros et al. 2013).

2-Ros et al. (2013) mentioned that the seven taxa; Didymodon rigidulus var. brevifolius Zodda, D. tophaceus fo. lingulatus (Boulay) Mönk., D. tophaceus var. cucullatus Bott., Gymnostomum calcareum var. obtusum Boulay, Hyophila pampanini Zodda, Hypnum cyrenaicum Müll. Hal. ex E. Durand \& Barratte and Tortella nitida var. obtusa (Boulay) Jelenc, were reported from Libya but "their identities have not been revised since", therefore, not included in our present list.

3-Publications based on the recent moss collections (made between 2004 and 2008) resulted in the report of 39 taxa; 14 of which represented new records published between 2006 and 2017 while 25 confirmed the existence of old records (given the symbol $\square$ in the present list).

It is expected that the investigation of the remaining part of the recent collections will result in many new records to the Libyan moss flora as well as confirm many of its old 


\section{Bryophytes of Libya. II. Musci: an annotated checklist}

records. The present article will, however, save all other future workers the time and effort they would have to spend on searching for the old, scattered and not easy to find publications dealing with the Libyan bryoflora; they can build up directly basing on the data compiled in the present checklist.

\section{List of Synonyms}

Synonyms given below are only those mentioned in publications related to Libya (based on Bottini, 1914, Pampanini, 1917, 1931, Bizot, 1973, Ros et al. 1999 and Ros et al. 2013 ).

Aloina ericaefolia (Lindb.) Kindb. nom. illeg. incl. spec. prior. = Aloina ambigua (Bruch\& Schimp.) Limpr. Aloina rigida var. ambigua (Bruch \& Schimp.) E.J. Craig = Aloina ambigua (Bruch \& Schimp.) Limpr. Aloina stellata Kindb. nom. illeg. incl. spec. prior. = Aloina rigida $(\mathrm{Hedw}$.$) Limpr.$

Aloina stellata var. pilifera (de Not.) Schiffn. = Aloina bifrons (De Not.) Delgad.

Anisothecium rubrum (Huds.) Lindb. nom. illeg. incl. spec. prior. = Dicranella varia (Hedw.) Schimp. Anisothecium rubrum var. tenuifolium (Bruch \& Schimp.) Braithw. = Dicranella howei Renauld \& Cardot Anisothecium varium (Hedw.) Mitt. = Dicranella varia (Hedw.) Schimp.

Barbula acuta (Brid.) Brid. = Didymodon acutus (Brid.) K. Saito

Barbula fallax Hedw. = Didymodon fallax (Hedw.) R.H. Zander

Barbula fallax var. brevicaulis (Schwägr.) Huebener = Didymodon fallax (Hedw.) R.H. Zander

Babula fiorii (Venturi) G. Roth = Tortula revolvens (Schimp.) G. Roth

Barbula hornschuchiana Schultz = Pseudocrossidium hornschuchianum (Schultz) R.H. Zander

Barbula nitida $($ Lindb.) Jur. = Tortella nitida $($ Lindb.) Broth.

Barbula revoluta Brid. = Pseudocrossidium revolutum (Brid.) R.H. Zander

Barbula revolvens Schimp. $=$ Tortula revolvens (Schimp.) G. Roth

Barbula rigidula (Hedw.) Mitt. $=$ Didymodon rigidulus Hedw.

Barbula vinealis Brid. $=$ Didymodon vinealis (Brid.) R.H. Zander

Barbula vinealis var. cylindrica (Taylor) Boulay nom. illeg. = Didymodon insulanus (De Not.) M.O. Hill

Brachythecium populeum (Hedw.) Schimp.=Sciuro-hypnum populeum (Hedw.) Ignatov \& Huttunen

Bryum bicolor Dicks. = Bryum dichotomum Hedw.

Bryum bicolor var. dolioloides $($ Solms) Warnst. $=$ Bryum dichotomum Hedw.

Bryum capillare var. meridionale Schimp. = Ptychostomum capillare (Hedw.) Holyoak \& N. Pedersen

Bryum capillare var. platyloma (Schwägr.) Schimp. = Ptychostomum capillare (Hedw) Holyoak \& N. Pedersen

Bryum capillare var. rufifolium (Dixon) Podp. = Ptychostomum capillare (Hedw.) Holyoak \& N. Pedersen

Bryum donianum Grev. = Ptychostomum donianum (Grev.) Holyoak \& N. Pedersen

Bryum murale Wilson ex Hunt. hom. illeg. = Bryum radiculosum Brid.

Bryum obconicum Hornsch. = Ptychostomum boreale (F. Weber \& D. Mohr) Ochyra \& Bednarek-Ochyra

Bryum pallescens Schleich. ex Schwägr. = Ptychostomum boreale $($ F. Weber \& D. Mohr) Ochyra \& Bednarek-Ochyra

Bryum torquescens Bruch \& Schimp. = Ptychostomum torquescens (Bruch \& Schimp.) Ros \& Mazimpaka

Camptothecium aureum (Spruce) Schimp. = Homalothecium aureum (Spruce) H. Rob.

Ceratodon chloropus (Brid.) Brid. = Cheilothela chloropus (Brid.) Broth.

Crossidium griseum (Jur.) Jur. = Crossidium squamiferum (Viv.) Jur. var. pottioideum (De Not) Mönk.

Crossidium squamigerum (Viv.) Jur. nom. inval. err. orthogr. = Crossidium squamiferum (Viv.) Jur.

Dicranella varia var. tenuifolia (Bruch \& Schimp.) Schimp. = Dicranella howei Renauld \& Cardot

Didymodon rigidulus var. gracilis (Schleich. ex Hook. \& Grev.) R.H. Zander= Didymodon acutus (Brid.) K. Saito

Didymodon tophaceus var. acutifolius Limpr. nom. illeg. incl. var. prior. = Didymodon tophaceus (Brid.) Lisa

Drepanocladus kneiffii (Schimp.) Warnst. = Drepanocladus aduncus (Hedw.) Warnst.

Encalypta rhaptocarpa var. trachymitria (Ripart) Wijk \& Margad. = Encalypta rhaptocarpa Schwägr. var. leptodon Lindb.

Encalypta vulgaris var. mutica Brid. = Encalypta vulgaris Hedw.

Entosthodon subpallescens Müll. Hal. nom. inval. = Entosthodon angustifolius Jur. \& Milde

Eucladium verticillatum var. angustifolium Lindb. $=$ Eucladium verticillatum (With.) Bruch \& Schimp.

Eurhynchium circinatum (Brid.) Schimp. $=$ Scorpiurium circinatum (Bruch) M. Fleisch. \& Loeske

Eurhynchium pumilum (Wilson) Schimp. = Microeurhynchium pumilum (Wilson) Ignatov \& Vanderp.

Fissidens cyprius Jur. = Fissidens crispus Mont.

Fissidens incurvus Starke ex Röhl.) Waldh. = Fissidens viridulus (Sw. ex Anon.) Wahlenb. var. incurvus (Starke ex Röhl.) Waldh.

Fissidens incurvus var. tamarindifolius (Turner) Braithw. = Fissidens viridulus (Sw. ex Anon.) Wahlenb. var. incurvus (Starke ex Röhl.) Waldh.

Fissidens limbatus Sull. $=$ Fissidens crispus Mont.

Fissidens mouretii Corb. $=$ Fissidens crassipes Wilson ex Bruch \& Schimp. subsp. warnstorfii (M. Fleisch.) Brugg.-Nann.

Funaria dentata Crome $=$ Entosthodon muhlenbergii (Turner) Fife

Funaria hygrometrica var. calvescens (Schwägr.) Mont. = Funaria hygrometrica Hedw.

Funaria mediterranea Lindb. = Entosthodon muhlenbergii (Turner) Fife

Funaria muhlenbergii Turner $=$ Entosthodon muhlenbergii (Turner) Fife

Funaria hygrometrica var. calvescens (Schwägr.) Mont. = Funaria hygrometrica Hedw.

Gymnostomum calcareum var. muticum Boulay = Gymnostomum calcareum Nees \& Hornsch.

Hypnum cupressiforme var. tectorum Brid. = Hypnum cupressiforme Hedw. var. lacunosum Brid.

Leptodon smithii var. filescens Renauld nom. nud. = Leptodon smithii (Hedw.) F. Weber\& D. Mohr

Oxyrrhynchium pumilum (Wilson) Loeske = Microeurhynchium pumilum (Wilson) Ignatov \& Vanderp.

Oxyrrhynchium rusciforme (Neck.) Warnst. = Rhynchostegium riparioides (Hedw.) Cardot 
Phascum cuspidatum var. piliferum (Hedw.) Hook. \& Taylor = Tortula acaulon (With.) R.H.Zander var. pilifera (Hedw.) R.H. Zander Phascum piliferum Hedw. = Tortula acaulon (With.) R.H.Zander var. pilifera (Hedw.) R.H. Zander Platyhypnidium rusciforme M. Fleisch. nom. illeg. incl. spec. prior. = Rhynchostegium riparioides (Hedw.) Cardot Platyhypnidium rusciforme var. atlanticum (Brid.) Machado-Guim. = Rhynchostegium riparioides (Hedw.) Cardot Pleurochaete squarrosa (Brid.) Lindb. = Tortella squarrosa (Brid.) Limpr.

Pottia commutata Limpr. = Microbryum davallianum (Sm.) R.H. Zander

Pottia davalliana (Sm.) C.E.O. Jensen = Microbryum davallianum (Sm.) R.H. Zander

Pottia starckeana (Hedw.) Müll. Hal. = Microbryum starckeanum (Hedw.) R.H. Zander

Pottia starckeana var. brachyodus (Bruch \& Schimp.) Müll. Hal = Microbryum starckeanum (Hedw.) R.H. Zander

Rhynchostegium rusciforme (F.W. Weiss ex Brid.) Schimp. nom. illeg. = Rhynchostegium riparioides (Hedw.) Cardot

Syntrichia intermedia Brid. = Syntrichia montana Nees

Timmiella barbula Limpr. nom. illeg. = Timmiella barbuloides (Brid.) Mönk.

Tortula fiorii (Venturi) G. Roth = Tortula revolvens (Schimp.) G. Roth

Tortula laevipila (Brid.) Schwägr. = Syntrichia laevipila Brid.

Tortula montana (Nees) Lindb. hom. illeg. = Syntrichia montana Nees

Tortula muralis var. incana (Bruch \& Schimp.) Wilson = Tortula muralis Hedw.

Tortula muralis var. obcordata (Schimp.) Limpr. = Tortula muralis Hedw.

Tortula revolvens var. obtusata Reimers $=$ Tortula revolvens (Schimp.) G. Roth

Trichostomum barbula Schwägr. nom. illeg. = Timmiella barbuloides (Brid.) Mönk.

Trichostomum brachydontium subsp. mutabile (Bruch) Giacom. = Trichostomum brachydontium Bruch

Trichostomum convolutum Brid. = Tortula atrovirens $(\mathrm{Sm}$.$) Lindb.$

Trichostomum inflexum Bruch = Tortella inflexa (Bruch) Broth.

Trichostomum nitidum (Lindb.) Schimp. = Tortella nitida (Lindb.) Broth.

\section{Acknowledgements}

All thanks, are do you to Prof. Dr. Rosa Ros of Murcia University in Spain for kindly providing us with relevant references and to Dr. Mai Taha of Ain Shams University for kind help.

\section{References}

Arts, T., Crundwell, A.C. and Whitehouse, H.L.K. (1995). Bryum valpairesense Thèr. an earlier name for B. pyriferum Crundw. and Whiteh. Journal of Bryology 18: 797-80.

Ascherson, P. (1881). Plantarum Africae septentrionalis mediae hucusque cognitarum conspectus. Botanisches Centralblatt 8: 27 (Cf. Ros, et al., 1999).

Bizot, M. (1973). Mouses africaines récoltées par M. Dénes Balázs. Acta Botanica Academiae Scientiarum Hungaricae 18: 7-28.

Bottini, A. (1914). Muschi di Libia. Bollettino di Studi ed Injormazioni del $R$. Giardino Coloniale. Palermo 1: 1-7.

Gallego, M.T., Cano, M.J., Ros, R.M. and Guerra, J. (1999). The genus Aloina (Pottiaceae, Musci) in the Mediterranean region and neighbouring areas. Nova Hedwigia 69 (1-2): 173-194.

Khalil, M. I. and Youssef, S.G.M. (2017). New Bryaceae records to the moss flora of Libya. Egypt. J. Exp. Biol. (Bot.) 13: 459-466.

Ochi, H. (1972). A revision of African Bryoideae, Musci (First part). Journal of the faculty of education, Tottori university, natural science 23: 1-126.

Pampanini, R. (1917). Plante di Bengasi e del suo Territorio raccolte dal Rev. P.D. Vito Zanon della Missione del PP. Giuseppini al Fuehat. Nuovo Giornale Botanico Italiano 24: 113- 118, 170-171.

Pampanini, R. (1931). Prodromo della Flora Cirenaica. Forli, Bryophyta, 69-81.
Ros, R.M., Cano, M.J. and Guerra, J. (1999). Bryophyte checklist of Northern Africa. Journal of Bryology 21: 207-244.

Ros, R.M., Mazimpaka, V., Abou-Salama, U., Aleffi, M, Blockeel, T. L., Brugués, M., C., Ros, R.M., Dia M. G., Dirkse G. M., Draper, El-Saadawi, W., Erdag, A., Ganeva, A., Gabriel, R., GonzálezMancebo, J. M., Granger, C., Herrnstadt, I., Hugonnot, V., Khali,I K., Kürschner, H., Losada-Lima, A., Luís, L., Mifsud, S., Privitera, M., Puglisi, M., Sabovljeić, M., Sérgio, C., Shabbara, H.M., SimSim, M., Sotiaux, A., Tacchi, R., Vanderpoorten, A., Werner, O. (2013). Mosses of the Mediterranean, an annotated checklist. Cryptogamie, Bryologie 34 (2): 99-283

Rungby, S. (1962). A contribution to the bryophytic flora of Spain and Morocco, especially the area between Gandia and Alcoy. Botaniska Notiser 115: 6164.

Shabbara, H.M. and Ghanem S.Y. (2006). Fabronia Raddi (Musci) in Libya. Egyptian journal of Biology 8: 100-105.

Youssef, S.G.M. (2017). Bryophytes of Libya. I. Hepatophyta: an Annotated Checklist. Egypt. J. Bot., 57(2): 277- 279.

Youssef, S.G.M., Abd-el-razik Khaled, S. and Hamad, R.B. (2009a). Zygodon Hook. ex Taylor a new record to Libya. Pakistan journal of Biological sciences 12 (24): 1571-1575.

Youssef, S.G.M., Abd-el-razik Khaled, S. and Hamad, R.B. (2009b). Orthotrichum schimperi Hammar a new record to Libya. Research journal of forestry 3 (4): 152-158.

Youssef, S.G.M., Khalil, M. I., Shabbara, H. and ElSaadawi, W. (2017a). Seven Brachytheciaceae (Musci) species new to Libya. Egypt. J. Bot., 57 (3): 605-919.

Youssef, S.G.M., Khalil, M. I., Shabbara, H. and ElSaadawi, W. (2017b). New Pottiaceae records to the moss flora of Libya. Egypt. J. Bot., (in press). 